\title{
Stellar Models: What is the future direction?
}

\author{
Asif ud-Doula \\ Penn State Worthington Scranton, Dunmore, PA 18512, USA \\ email: asif@psu.edu
}

\section{Introduction}

This was one of two general discussions at the conference and it focussed primarily on stellar modelling. In particular, we were interested in the quality of the models, how we can check the models and what the direction of future modelling will be given the rise in popularity of MESA.

Since there are no transcripts of the discussions available, the actual words of the speakers are paraphrased here briefly. In what follows I summarize the discussion in several separate themes which might or might not have occurred in the same order during the lively discussion session.

\section{Discussion \\ Quality of Stellar Models}

UD-Doula: Over the past few days we have been talking a lot about stellar evolution and modelling in general. Let us begin our discussion with how good our models are. Are they self-consistent?

Chieffi: A stellar evolutionary code is a very complex tool that should be used only by those who actively contribute to write it. It is very easy and extremely dangerous to simply push a button and get a result because there are so many choices and things that must always be kept in mind when computing even the simplest model that only an expert can really (try to) understand the meaning. It should be evident to everyone that different codes very often give different results, and since it is not easy at all to understand where such differences come from, only a comparison among similar computations obtained with totally different codes can really give us an idea of the robustness of a prediction, i.e that at least that there are no hidden numerical mistakes. For this reason I consider extremely dangerous the growing use of an open source stellar evolutionary code. Everyone can modify and compute without any control and this means that sometimes models may come out computed after having made nonsense changes to the code. No one can control this and since it is much easier to push a button instead of spending hours, weeks, years coding in front of a terminal, in the future I think that most of the people will just use such codes with the final total loss of knowledge (understanding) and control in this field. It is already evident with tools like MESA which are now being used like black boxes.

NoELs: I fully agree with Alessandro. According to Bill Paxton's own words MESA is just a tool, not a theory, a very convenient and efficient tool indeed but it should be not used as a black box. Consistency has to be checked, especially when models are used to interpret asteroseismic observations. It should be recalled that asteroseismology help bring some constraints on the chemical profile, not directly on the physical processes that have led to such a profile (see Andreas talk). An essential prerequisite is thus to have a fully consistent model in the frame of the physics adopted in the computation. 
During previous sessions in this meeting, we already discussed some problems related to the boundary of convective cores in massive stars (see also my talk for more details). An easy check is to verify that the radiative temperature gradient is equal to the adiabatic temperature gradient on the convective side of the boundary. If this is not the case, the position of the boundary is not correct and the inferences that will be brought from an asteroseismic analyses will be unreliable. This problem is mainly encountered in core helium burning models computed with the Schwarzschild criterion. As a result of helium burning, a discontinuity in helium abundance forms at the convective core boundary. If the equality of the temperature gradients is imposed on the radiative side of the boundary, convective neutrality is not met on the convective side. The convective core is then kept from growing, as it should normally do, and since the discontinuity in helium abundance increases with time, the incoherency becomes larger and larger. In red clump stars, this leads to a clear disagreement between seismic observations and theoretical models (see, e.g., Montalbán et al. 2013). With the Ledoux criterion the problem is already encountered in main-sequence models since a discontinuity in $\mu$-gradient exists at the core boundary. When the boundary is searched for through an interpolation process, notwithstanding the presence of such a discontinuity, convective cores are too small, have inconsistent boundaries, and are surrounded by apparently semi-convective layers, which should indeed be part of the convective cores if the location of the core boundary were properly determined.

Chitffi: The difference between the size of the 'theoretical' convective cores and the ones derived by the analysis of the seismic data is usually interpreted in terms of "overshooting", like if a good or a bad match between the theoretical and 'observed' values would deny/prove the presence of the physical phenomenon 'overshooting'. What is worst is that in all (most) papers that address such a comparison, the size of the convective core is not mentioned at all, the comparison is directly expressed in in terms of $\alpha$ (the free parameter that multiply the pressure scale height in the standard Mixing Length Theory). This approach assumes that each stellar code provides the same standard size of the convective core and that such a size is a firm theoretical prediction. This is false. The size of the convective core depends on both numerical and physical choices that may vary with time and from author to author. For example in the 80's the size of the 'overshooted region' was much larger than presently adopted just because the old opacity tables lead to smaller standard convective cores. Moreover a 'possible' extra mixing could be due to different physical mechanisms (e.g. rotation). Hence in my opinion the word overshooting should be totally dropped in this context and the differences should be discussed simply in terms of 'discrepancies/differences' between stellar models and real stars. The papers that address the comparison between theoretical and 'observed' convective cores should explicitly mention and publish the sizes of their standard convective cores and their chosen 'extended' ones in solar masses (not in terms of $\alpha$ ). Only in this way these data could be really fruitful for the community of the stellar modelers.

MORAVVEJI: We fortunately live in an era of ultra high-precision space photometry, but the question is whether or not stellar models are accurate enough to explain such observations. Currently, there are several flavours of stellar structure and evolution codes available, MESA being just one of them. I believe it is very timely for code developers to set up benchmarking exercises, to gauge the theoretical uncertainties on our best models, yet keeping the flavour of different codes. Such an exercise was already performed in 2008 in preparation for the analysis of CoRoT asteroseismic data (Lebreton et al. 2008). With 
a much improved frequency resolution from the Kepler 4-year observations, we still need to organize comparison and benchmarking exercises to improve our theoretical models.

PULS: I agree that the cross-checks are important and I am willing to take on this task. However, having enough time in hand is the problem. But I too caution against using black boxes. You need to talk to experts before using these tools.

AERTS: But talking to experts takes time, students need to get out the work. Can they afford it?

HERRERO: I think we should continue to use codes like MESA for estimates and to get a feeling of the physics involved, or even take the results when we are using well stablished conditions. But when we are using new physics or results or deriving important consequences we should ask the experts to understand their limitations. But I certainly believe we should not be using any code, whether for evolutionary, atmospheric or any other kind of models, as black boxes.

TKACHENKO: I agree with Alessandro's earlier comment that junior scientists should rely on senior researchers, as they are the ones who can understand the meaning better when computing even the simplest model. But even then one should always learn from experts to gain the necessary expertise otherwise there will be no experts in stellar modelling left anymore in 20-30 years from now, when all current experts retire!

Chieffi: As a final remark about self-consistency, let me say that this is something that cannot be fixed once for ever but it must be checked continuously every time the code or some physical ingredient (EOS, opacity, etc.) is changed. Some skill and experience is necessary to do that.

\section{Mass Loss Rates}

SUNDQVIST: Remember that high-mass stars can also have cool surfaces. The RSG case presumably needs 3-D, and especially with respect to their stellar winds RSGs have not been much investigated so far. Considering the importance of mass loss in this stage for the late evolution and fate of massive stars, this is an area where some big efforts seem to be quite urgently needed.

KHALACK: Analysis of stellar spectra obtained in the UV, visual and IR spectral regions provides information about physical conditions in stellar atmosphere and stellar wind (if it is strong enough). Each star is unique and its spectrum has some particular features, but one can always trace general tendencies (e.g., chemical peculiarity, presence of magnetic field, strong emission lines, P-Cyg profiles, etc.) that together with estimates of effective temperature and surface gravity might help identify the type of star and its stage of evolution. However, it would be useful if models provided more information (predictions) about phenomena observed during certain stages of stellar evolution in outer parts of stars that can be studied by the methods of spectral analysis, photometry and polarimetry. In this way one could verify a validity of model predictions and treat properly the observed particular features in stellar spectra (e.g., presence of weak emission lines, line profile asymmetry, abundance stratification, wind clumping, etc.) employing more realistic physical conditions in stellar atmosphere and/or wind of studied star. 


\section{Dimensionality}

VINK: The majority $(>80 \%)$ of massive stars have large-scale winds that are more or less spherical, so here 1D is not too bad an approximation (though 2D needs to be considered for the exceptions). However, because winds are intrinsically clumpy, 3D effects are still needed in the end. And these small-scale 3D effects may have crucial large-scale consequences!

SUNDQVIST: I think it is important to consider also the outermost layers in cases where radiation pressure dominates the support against gravity. Several radiation-hydrodynamic instabilities are expected when $\Gamma \rightarrow 1$, presumably leading to a highly structured atmosphere. So multi-dimensionality and corresponding simulations are probably key here.

Mathis: Most of the processes that drive transport of angular momentum and chemicals mixing in stellar interiors are 3D (see e.g. hydrodynamical and MHD instabilities and turbulence, internal gravity waves, etc., e.g. Brun (2011) and the review by Meakin (2008)). Today, we are able to simulate them on dynamical time-scales using LES for global geometry and DNS in local boxes. However, these 3D non-linear simulations that use super-calculators have a large computation time for one star and cannot yet be computed for evolution time-scales. Therefore, to tackle the effects of 3D MHD mechanisms on stellar evolution, it is necessary to extract prescriptions and scaling laws that can be implemented in 1- or 2-D stellar evolution codes that treat the large-scale transport of angular momentum on secular time-scales along the whole evolution of stars (e.g. Meynet \& Maeder 2000; Mathis et al. 2013; Rieutord \& Espinosa 2013). Moreover, these "secular" dynamical stellar evolution codes are those that allow today to compute the grids of stellar models necessary to interpret the large amount of data coming for example from asteroseismology, spectroscopy, and spectropolarimetry.

VINK: There is sometimes still a tendency in the community to be happier with detections of magnetic field rather than with non-detections (i.e. detections get published, and non-detections do not) whether or not this is about wind asphericity or magnetic field incidence. But the point is that non-detections can scientifically be as interesting as detections and we need to discuss ramifications of such non-detections more broadly.

\section{References}

Brun, A. S. 2011, in H. Wozniak \& G. Hensler (eds.), EAS Publications Series, Vol. 44 of EAS Publications Series, pp 81-95

Lebreton, Y., Montalbán, J., Christensen-Dalsgaard, J., Roxburgh, I. W., \& Weiss, A. 2008, ApESSS 316, 187

Mathis, S., Decressin, T., Eggenberger, P., \& Charbonnel, C. 2013, A\& A 558, A11

Meakin, C. A. 2008, in L. Deng \& K. L. Chan (eds.), IAU Symposium, Vol. 252 of IAU Symposium, pp 439-449

Meynet, G. \& Maeder, A. 2000, A\&̈A 361, 101

Montalbán, J., Miglio, A., Noels, A., et al. 2013, ApJ 766, 118

Rieutord, M. \& Espinosa, L. F. 2013, in L. Cambresy, F. Martins, E. Nuss, \& A. Palacios (eds.), SF2A-2013: Proceedings of the Annual meeting of the French Society of Astronomy and Astrophysics, pp 101-104 